\title{
COMPARISON OF DATA-DRIVEN RECONSTRUCTION METHODS FOR FAULT DETECTION
}

\author{
P. Baraldi ${ }^{1}$, F. Di Maio ${ }^{1}$, D.Genini ${ }^{1}$, E. Zio ${ }^{1,2}$ \\ ${ }^{I}$ Energy Department, Politecnico di Milano, Via Ponzio 34/3, 20133 Milano, Italy \\ piero.baraldi@polimi.it \\ ${ }^{2}$ Chair on System Science and the Energetic Challenge, European Foundation for New \\ Energy - Paris and Supelec, Paris, France
}

\begin{abstract}
This work proposes a comparison of three data-driven signal reconstruction methods, i.e. AutoAssociative Kernel Regression (AAKR), Fuzzy Similarity (FS) and Elman Recurrent Neural Network (RNN), for fault detection based on the difference between the signal observations and the reconstructions of the signal in normal condition. The aim is to show the capabilities and drawbacks of the methods and propose a strategy for the aggregation of their outcomes, in order to overcome their limitations. For this purpose, the performance of each method is evaluated in terms of fault detection capability, considering accuracy, robustness and resistance to the spillover effect of the obtained signal reconstructions. The comparison is supported by the application to a real industrial case study regarding temperature signals collected during operation of a rotating machine in an energy production plant. An ensemble of the three methods is proposed to overcome the limitations of the three methods.
\end{abstract} Formattato: Non Evidenziato

Keywords - fault detection, signal reconstruction, Auto-Associative Kernel Regression (AAKR), Fuzzy Similarity (FS), Elman Recurrent Neural Network (RNN), Ensemble of methods. 


\section{Introduction}

Accurate monitoring of the health state of Systems, Structures and Components (SSCs) can

Formattato: Non Evidenziato contribute significantly to the safe and efficient functioning of power plants, assuring timely detection of malfunctions and anomalies during operations. In particular, fault detection allows to determine whether faults are present and to localize them early in their development [Ma et al., 2011].

In order to prevent over/under estimation of anomalous conditions, a desideratum for fault detection methods is the reliability of their residuals estimates [Far et al., 2009]. In practice, two phenomena may occur if the signal reconstruction is not reliable: i) false alarms in case of over-estimation of anomalous conditions and ii) missing alarms in case of under-estimation of anomalous conditions [Chetouani, 2006; Arinton et al., 2012; Di Maio et al., 2013].

Typically, fault detection algorithms compare the signal values measured during operation with those estimated (reconstructed) by a model as if the system were in normal conditions. The calculated residuals between observed and reconstructed values reveal the presence of anomalous conditions [Reifman, 1997]. Signal reconstruction can be accomplished through a variety of methods that can be classified into analytical and empirical, where the former are based on the knowledge of the physical equations describing normal and anomalous conditions, whereas the latter are trained using signal measurements collected during the range of operating conditions of the plant [Hines et al., 2008; Baraldi et al., 2011; Di Maio et al., 2013].

Analytical methods are applied when the underlying mechanism of a system is well understood, and can be used to reconstruct its expected normal behavior based on the measured signals. The application of these methods in large and complex systems is limited due to the large efforts necessary for the development of the analytical models, especially when the involved phenomena are not well understood [Coble et al., 2012].

Recently, the increasing availability of large datasets of signal measurements has been favoring the use of empirical rather than analytical methods for signal reconstruction [Coble et al., 2012]. 
Empirical methods do not require an explicit understanding of the underlying physical mechanism of the system [Hines et al., 2008; Zhao et al., 2013]. They are classified according to two characteristics: parametric versus non parametric and inferential versus auto-associative [Coble et al., 2012]. Parametric methods aim at building the best fitting of signal values using the present and historical available information to reconstruct new observations. On the other hand, non-parametric methods combine present and historical data through an algorithm to reconstruct each new observation. Inferential methods concentrate on a set of explanatory signals to reconstruct another signal values [Zhang et al., 2011]. On the contrary, auto-associative methods use a set of measured signals to provide the reconstruction of the same signal values. Among these categories, several empirical methods have been developed. Typical examples include Artificial Neural Networks (ANNs) [Hines et al., 1996] and Recurrent Neural Networks (RNNs) [S. Seker et al., 2003], Principal Component Analysis (PCA) [Hines et al., 2008; Luh et al., 2011] and Independent Component Analysis (ICA) [Al-Bazzaz et al., 2004], Auto-Associative Kernel Regression (AAKR) [Garvey et al., 2006; Baraldi et al., 2012], Multivariate State Estimation Technique (MSET) [Zavaljevski et al., 2000], Support Vector Machines (SVMs) [Zavaljevski et al., 2000; Zio et al., 2012] and Fuzzy Similarity (FS) [Zio et al., 2010a].

Although a large number of signal reconstruction methods has been developed, comparative analyses of their performance when applied to real industrial case studies have seldom been proposed in practical settings and guidelines for the choice of the adequate performing methods of fault detection in different settings have not been provided.

In the present work, we propose a comparison of three signal reconstruction methods used for fault detection, i.e. AAKR, FS and Elman RNN. The objective is to compare their performances in different situations with respect to general fault detection targets: fast detection speed, and low false and missing alarms rates [Wang et al., 2005]. A real case study concerning the monitoring of a rotating machine in an energy production plant has been considered for the comparison study. Furthermore, a strategy for the aggregation of the individual outcomes of AAKR, FS and Elman 
RNN is proposed in order to overcome the limitations of the three independent reconstruction methods by resorting to an ensemble strategy.

The remainder of the paper is organized as follows: Section 2 describes the problem, Section 3 illustrates the signal reconstruction methods employed and Section 4 shows their application to a case study regarding the signals collected during the operational transients of a rotating machine. Finally, Section 5 introduces the ensemble of the three methods and Section 6 draws the conclusions.

\section{Problem statement}

We consider a training set $\overline{\bar{X}}^{t r}$ containing the values of $J$ signals measured during normal plant conditions at $N$ different time instants. The generic element $x^{t r}(k, j)$ of $\overline{\bar{X}}^{t r}$ indicates the value of signal $j$, with $j=1, \ldots J$ and $k=1, \ldots, N$.

The objective of a signal reconstruction method is to provide the reconstruction, $\hat{x}(t, j)$, of the value the $j$-th signal observed at time $t, x(t, j)$. Then, the deviation (residual), $r(t, j)=\hat{x}(t, j)-x(t, j)$, between the signal reconstruction and the observation can be compared with a properly defined threshold $d$, and if $r(t, j)>d$ an anomalous condition is identified [Yu et al., 2006]. The threshold $d$ is typically fixed considering the reconstruction error on a validation set

Codice campo modificato

Codice campo modificato

Codice campo modificato

Codice campo modificato

Codice campo modificato

Codice campo modificato

Codice campo modificato

Formattato: Non Evidenziato made by measurements performed at $N_{v a l}$ time instants, different from those of the training set. In the present work, the threshold for the residuals of the $j$-th signal is set to:

$$
d(j)=4 \sqrt{\operatorname{MSE}(j)}
$$

where $\operatorname{MSE}(j)$ is the Mean Square Error (MSE) of the $j$-th signal residuals computed considering the validation set:

$$
\operatorname{MSE}(j)=\frac{\sum_{k=1}^{N_{v a l}}(\hat{x}(k, j)-x(k, j))^{2}}{N_{v a l}}
$$


Since the residuals are expected to be Gaussian-distributed with mean equal to 0 and variance $\operatorname{MSE}(j)$, a constant value equal to 4 has been used in Eq. (1) in order to reduce the false alarm rate and to guarantee the detection of the abnormal conditions when the residuals exceed process noise in normal conditions. Other advanced statistical techniques for the analysis of the residuals and the detection of abnormal conditions can be found in [Di Maio et al., 2013].

Three reconstruction methods are considered in the present work, i.e. AAKR, FS and RNN, and their performance is compared with respect to three metrics: accuracy, robustness and spillover. These metrics are computed considering a test set made by $N_{\text {test }}$ measurements different from those of the training and validation sets.

For the generic signal $j$, accuracy is the ability of providing correct reconstructions of observed data in normal conditions and is evaluated in terms of the global MSE on the test set:

$$
\operatorname{MSE}(j)=\frac{\sum_{k=1}^{N_{\text {test }}}(\hat{x}(k, j)-x(k, j))^{2}}{N_{\text {test }}}
$$

Robustness with respect to anomalous behavior is the ability of reconstructing the values of signal $j$ expected in normal conditions and is computed as the difference between the reconstruction of the $j$-th signal in anomalous conditions, $\hat{x}_{a c}(k, j)$, and the observation of the same signal in normal conditions, $x(k, j)$ :

$$
S(j)=\frac{\sum_{k=1}^{T}\left(\hat{x}_{a c}(k, j)-x(k, j)\right)^{2}}{T} .
$$

Therefore, a low value of $S(j)$ means high robustness [Baraldi et al., 2011]. When a real dataset containing real abnormal conditions measurement is not available for the computation of the metric in (4), the abnormal conditions are simulated by adding a random noise of fixed variance to historical normal conditions values.

A global robustness measure over all signals can be, finally, constructed as:
Formattato: Non Evidenziato

Formattato: Non Evidenziato

Formattato: Non Evidenziato

Formattato: Non Evidenziato

Formattato: Non Evidenziato

Formattato: Non Evidenziato

Formattato: Non Evidenziato

Formattato: Non Evidenziato

Formattato: Non Evidenziato

Formattato: Non Evidenziato

Formattato: Non Evidenziato

Formattato: Non Evidenziato

Formattato: Non Evidenziato 


$$
S=\sum_{j=1}^{J} S(j)
$$

Spillover measures the effect that the anomalous behavior of the monitored signal $j$ has on the reconstruction of the other signals. The spillover effect from signal $j$ can lead to incorrect model reconstructions of other signals. Thus, the accuracy $A$ in the reconstruction of signal $j_{1}$ in normal conditions when signal $j_{2}$ is in anomalous conditions is defined by:

$$
A\left(j_{1} \mid j_{2} \text { is anomalous }\right)=\frac{\sum_{t=1}^{N_{\text {test }}}\left(\hat{x}\left(k, j_{1}\right)-x\left(k, j_{1}\right)\right)^{2}}{N_{\text {test }}}
$$

A global spill-over measure over all signals can, then, be constructed as:

$$
A=\sum_{j_{1}=1}^{J} \frac{\frac{\sum_{j_{2} \neq j_{1}=1}^{J} A\left(j_{1} \mid j_{2} \text { is anomalous }\right)}{J-1}}{J}
$$

\section{Signal reconstruction methods}

The signal reconstruction methods AAKR, FS and Elman RNN are analyzed and compared in order to find $i$ ) in which situations it is appropriate to apply one method or another and $i i$ ) an ensemble of the three methods that overcomes the limitations of the methods. For completeness of the paper, a brief introduction on these methods is provided in Sections 3.1, 3.2 and 3.3, respectively.

\subsection{Signal reconstruction using AAKR}

The AAKR method provides the vector of reconstructed signal values, $\overline{\hat{x}}(t)=[\hat{x}(t, 1), \hat{x}(t, 2), \ldots, \hat{x}(t, J)], \quad$ given the current signal measurement vector, $\bar{x}(t)=[x(t, 1), x(t, 2), \ldots, x(t, J)]$, whereby each reconstructed value is the sum of the historical observations, $\overline{\bar{X}}^{t r}$, weighted by a Gaussian kernel [Baraldi et al., 2011]:

$$
w(k)=\frac{1}{\sqrt{2 \pi} h} e^{-\frac{d^{2}(k)}{2 h^{2}}}
$$

Codice campo modificato

Codice campo modificato 
The parameter $h$ defines the Gaussian bandwidth and $d^{2}$ is the Euclidean distance between the $J$ signal measurements $\bar{x}(t)$ and the $k$-th observation in $\overline{\bar{X}}^{t r}$ for every single pattern $k$ :

$$
d^{2}(k)=\sum_{j=1}^{J}\left(x(t, j)-x^{t r}(k, j)\right)^{2}
$$

Reconstruction, $\hat{x}(t, j)$, of the $j$-th signal, $x(t, j)$, is obtained by weighting on the weights $w(k)$ :

$$
\hat{x}(t, j)=\frac{\sum_{k=1}^{N} w(k) x^{t r}(k, j)}{\sum_{k=1}^{N} w(k)}
$$

\subsection{Signal reconstruction using FS}

The basic idea of the method is to compare the segment of a test trajectory containing only the most recent $L_{t}$ measurements of signal $j$ at the present time $t, \bar{x}_{t}(j)=x\left(t-L_{t}+1: t, j\right)$, and the generic segment of length $L_{t}$ of signal $j$ contained in the training set $\overline{\bar{X}}^{t r}$, which ends at time $k$, $\bar{x}^{t r}(k, j)=\bar{x}^{t r}\left(k-L_{t}+1: k, j\right)$, with $k=L_{t}, L_{t}+1, . ., T$, hereafter called reference trajectory [Baraldi et al., 2014]. The comparison is based on a fuzzy definition of trajectory pattern similarity proposed in [Zio et al., 2010a] in the context of fault prognostics.

More specifically, the pattern matching process is based on the evaluation of a fuzzy distance between the reference and test trajectory patterns [Angstenberger, 2001]; then, the reconstruction is obtained applying a fuzzy distance-weighted sum of the reference trajectories.

The distance evaluation is based on a pointwise difference $\delta^{2}(k, j)$ between the $L_{t}$ elements of the $k$-th trajectory $\bar{x}_{k}^{t r}(j)$ and the elements of the test trajectory $\bar{x}_{t}(j)$ of the $j$-th signal, given by:

$$
\delta^{2}(k, j)=\left|\bar{x}_{k}^{t r}(j)-\bar{x}_{t}(j)\right|^{2}
$$

The distances are finally aggregated into [Zio et al., 2010b]:

$$
\delta^{2}(k)=\frac{\sum_{j=1}^{J} \delta^{2}(k, j)}{J}
$$

Codice campo modificato

Codice campo modificato

Codice campo modificato

Codice campo modificato

Codice campo modificato

Codice campo modificato

Codice campo modificato

Codice campo modificato

Codice campo modificato

Codice campo modificato

Codice campo modificato

Codice campo modificato

Codice campo modificato

Codice campo modificato Codice campo modificato Codice campo modificato 
To account for a gradual transition between 'similar' and 'non-similar', we introduce an “approximately zero" fuzzy set [Zio et al., 2010c] taken, in this work, as a bell-shaped function:

$$
\mu(k)=e^{-\left(-\frac{\ln (\alpha)}{\beta^{2}} \delta^{2}(k)\right)}
$$

The parameters $\alpha$ and $\beta$ are set by the analyst: the larger the value of the ratio $\ln (\alpha) / \beta^{2}$, the narrower the fuzzy set and the stronger the definition of similarity [Zio et al., 2010a].

The distance score $d(k)$ between two trajectories is, then, computed as:

$$
d(k)=1-\mu(k)
$$

With respect to AAKR, weighted reconstruction allows all the reference training trajectories $\bar{x}^{t r}(k, j)$ (rather than only the single measurement $\left.x^{t r}(k, j)\right)$ carry useful information for the reconstruction of the missing data in the currently developing trajectory [Baraldi et al., 2014]. To this aim, weights are computed with a decreasing monotone function [Zio et al., 2010a], such that the smaller the distance $d(k)$ the larger the weight given to the $k$-th reference trajectory:

$$
w(k)=(1-d(k)) e^{-\frac{1}{\beta} d(k)} \quad k=L_{t}, \ldots, T
$$

The same value of $\beta$ used in eq. (13) is here employed in order to reduce the number of parameters to be set. Finally, the reconstruction, $\hat{x}(t, j)$, of the observed value at time $t$ of the $j$-th signal, $x(t, j)$ , in the test trajectory is the weighted sum of the last element $x^{t r}(k, j)$ of each reference trajectory [Baraldi et al., 2014]:

$$
\hat{x}(t, j)=\frac{\sum_{k=1}^{N-L_{t}} w(k) x^{t r}(k, j)}{\sum_{k=1}^{N-L_{t}} w(k)}
$$

\section{Codice campo modificato}

Codice campo modificato

Codice campo modificato

Codice campo modificato

Codice campo modificato

Codice campo modificato

Codice campo modificato

Codice campo modificato

Codice campo modificato

Codice campo modificato

Codice campo modificato

Codice campo modificato

Codice campo modificato

Codice campo modificato

Codice campo modificato 


\subsection{Signal reconstruction using RNN}

The architecture of the Elman RNN that has been used in this work consists in input, context, hidden and output layers which are connected by weights (synapses) [Seker et al., 2003]. This RNN is constructed in such a way that the outputs of some layers are fed back to the same or preceding layers [Gabrijel et al., 2003]. At a specific time $t$, the input at time $t-1$ and at current time $t$ are used as inputs to the network. Thus, the input layer entails also considering the input node (neuron) fed with the observed value at time $t-1$ of the $j$-th signal, $x(t-1, j)$. The output, $\hat{x}(t, j)$, results into the aggregation of previous and current observed values [Elman, 1990; Pham et al., 1996; Gabrijel et al., 2003; Seker et al., 2003]. The standard back-propagation learning rule is typically employed to train the network [Rumeihart et al., 1986].

Indicating by $u_{i}(t)$ the total input to the $i$-th hidden node, by $y_{i}(t)$ the output of the $i$-th hidden node and by $y_{k}^{c}(t)$ the output of the $j$-th context node, the following equations hold:

$$
u_{i}(t)=\sum_{k=1}^{n} w_{i, k}^{x} l(t-1) y_{k}^{c}(t, j)+w_{i}^{u}(t-1) x(t, j)
$$

$$
\begin{gathered}
x_{i}(t)=f\left(u_{i}\right) \\
y_{k}^{c}(t)=x(t-1, j) \\
\hat{x}(t, j)=\sum_{i=1}^{n} w_{i}^{y}(t-1) y_{i}(t)
\end{gathered}
$$

where $w_{i}^{u}, w_{i, k}^{x}$ and $w_{i}^{y}, i, k: 1,2, \ldots, n$, are the weights of the links between the input node and the hidden layer, between the context layer and the hidden layer, and between the hidden layer and the output node, respectively; $f$ is a sigmoidal activation function.

\section{Case study}

A real industrial case study concerning the identification of anomalous operational transients in a

Codice campo modificato

Codice campo modificato

Codice campo modificato

Codice campo modificato

Codice campo modificato

Codice campo modificato

Codice campo modificato

Codice campo modificato

Codice campo modificato 
due to confidentiality reasons) has been considered. A dataset containing the measurements of $J=5$ temperature signals taken for a total of $T=6000$ time instants during 1 year is available.

Formattato: Non Evidenziato Codice campo modificato

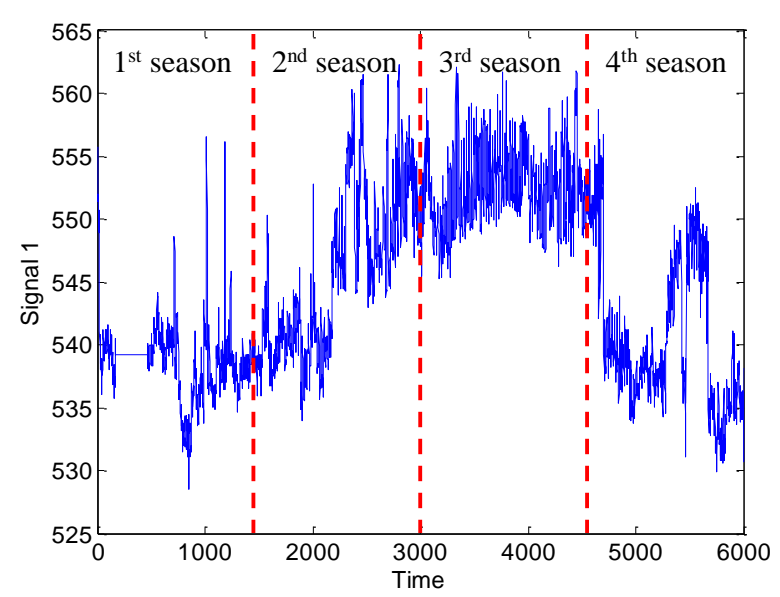

Figure 1. Evolution of signal 1 (name and units are purposely hidden, for confidentiality reasons). The red lines divide the data into 4 seasonal behaviors.

An extensive pre-analysis of the available dataset has highlighted a seasonal behavior of the signals, which is due to different operational/environmental conditions. Thus, we have divided the original dataset into 4 seasonal sets containing 1500 measurements each (as shown in Figure 1). In season 1, a constant temperature has been measured between $t=197$ and $t=591$ due to a problem of the measurement sensor.

A training set $\overline{\bar{X}}^{t r}$ has been built by taking the first 800 measurements of each set. The remaining 700 measurements in each season are equally divided into a validation set for optimally tuning the AAKR, FS and RNN parameters and setting the value of the threshold $d$ (see Section 2), and a test set for accuracy, robustness and spillover metrics evaluation.
Formattato: Non Evidenziato

Formattato: Non Evidenziato 
The parameters of the three reconstruction methods have been set by performing an automatic tuning procedure based on trial and error. In practice, with respect to the AAKR method, a possible range of values $[0.01,0.4]$ of the parameter $h$ has been identified, and the reconstruction of the validation set has been performed considering 40 equally spaced values. The minimum value of the MSE on the validation set has been obtained for $h=0.04$ (Figure 2).

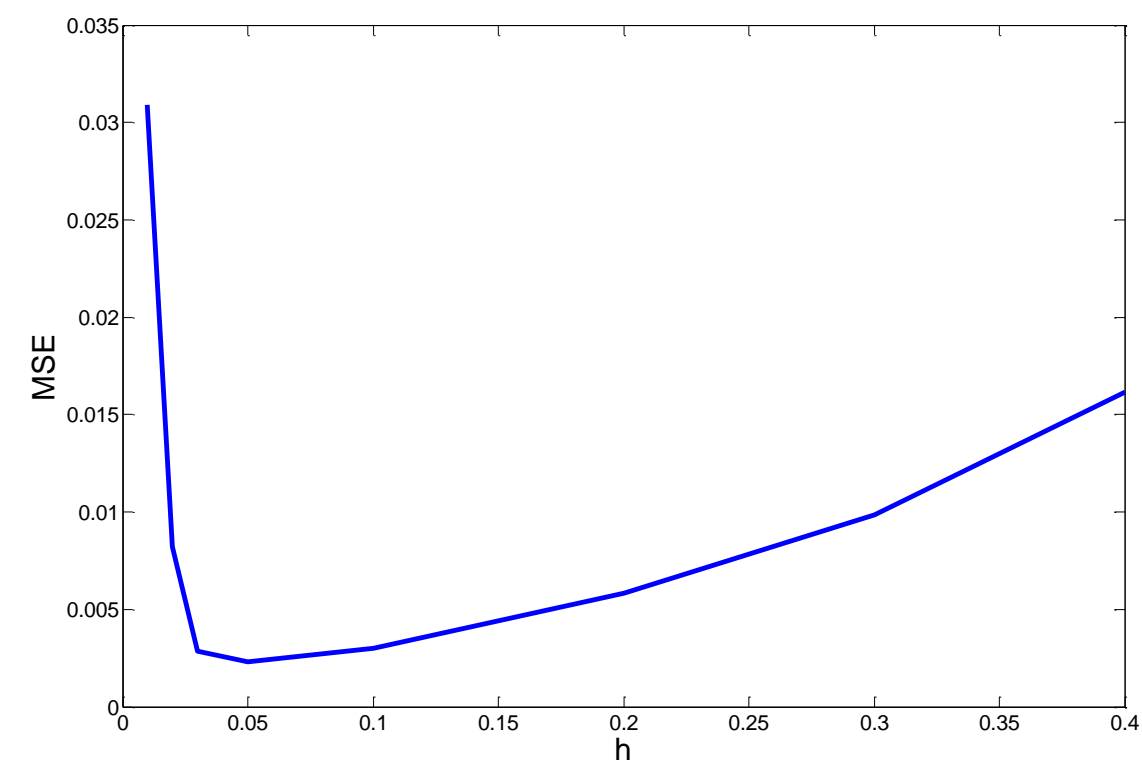

Figure 2. Evolution of the MSE on the reconstruction of the validation set with the AAKR parameter $h$.

Similarly, the three parameters, $\alpha, \beta$ and $L_{t}$, of the FS method have been set by considering a three dimensional grid with $\alpha, \beta$ and $L_{t}$ values in the ranges [0.005, 0.4], [0.01, 0.3] and [2,10], respectively. The $\left(\alpha, \beta, L_{t}\right)$ triplet with associated minimum MSE in the reconstruction of the validation set is $(0.05,0.05,2)$ (Figure 3). Finally, The RNN has required setting the number $k$ of nodes in the hidden layer. This has been done by developing RNNs with a number of hidden nodes
Formattato: Non Evidenziato

Formattato: Non Evidenziato

Formattato: Non Evidenziato

Formattato: Non Evidenziato 
from 6 to 20 and selecting the one with 14 nodes, since it gives the lowest MSE in the reconstruction of the validation set. Figure 4 shows the evolution of the MSE on the validation set as a function of the number $k$ of nodes.

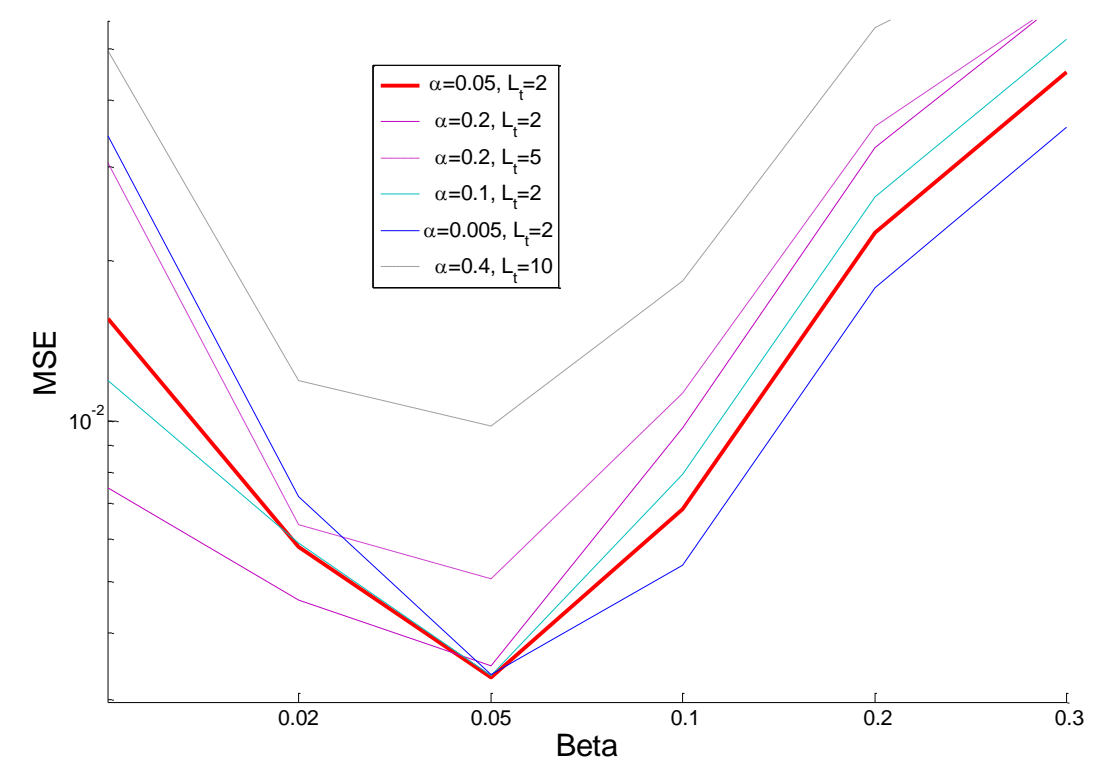

Formattato: Non Evidenziato

Formattato: Non Evidenziato

Figure 3. Evolution of the MSE on the validation set for the FS method. The x-axis reports different $\beta$ values, the line style corresponds to different $L_{t}$ values (continuous, dashed and dotted lines for $L_{t}=\mathbf{2 , 5}$ and 10, respectively). 


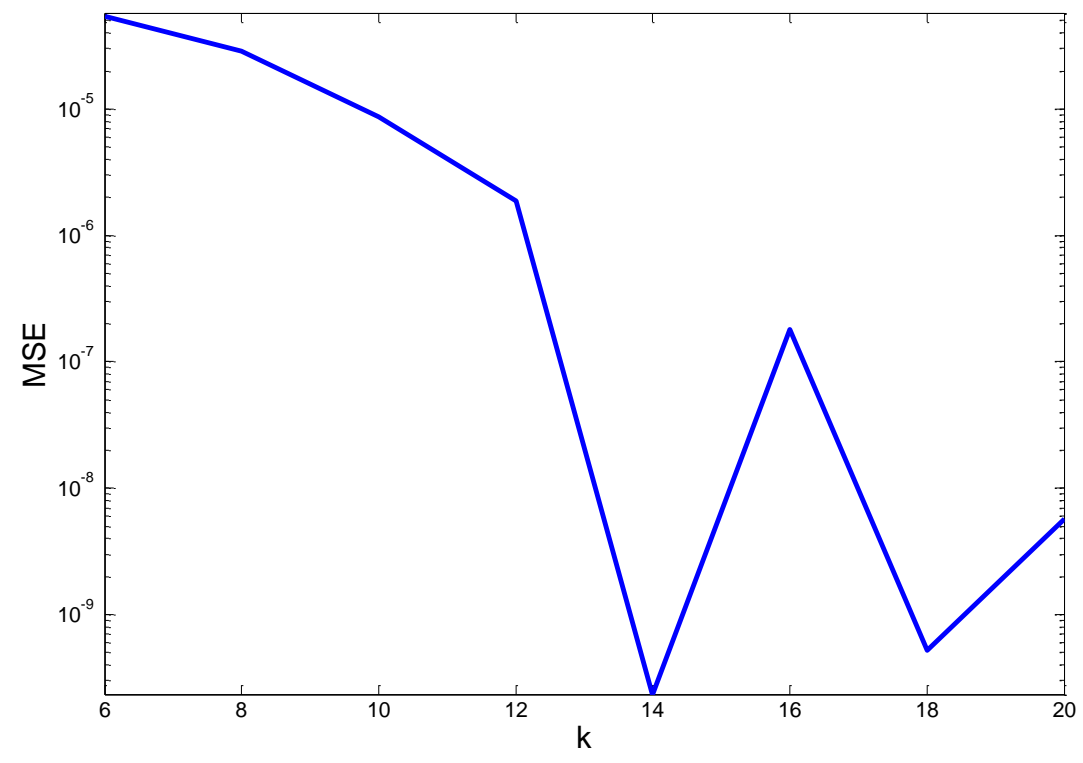

Figure 4. Evolution of the MSE on the reconstruction of the validation set patterns with the parameter $k$.

Although the obtained parameter values are specific for the application considered in this work, the proposed trial and error procedure for parameters setting can be adopted in other applications. The interested reader can refer to [Baraldi et al., 2011] for an application of the AAKR and the parameters setting procedure to the monitoring of a reactor coolant pump of a nuclear power plant. The application of the FS and the parameters setting procedure has been discussed in [Baraldi et al., 2014] with respect to the reconstruction of missing data in shut-down transients of a turbine. Application of the RNN and discussion of the parametesr setting can be found in [Gabrijel et al., 2003 ] in the context of on-line identification and reconstruction of finite automata.

\subsection{Accuracy}

Figure 5 shows the reconstruction residuals (continuous line) of the test set formed by 1600 values (400 measurements for each season) and the corresponding thresholds $d=4 \sqrt{\mathrm{MSE}}$ (dashed lines)

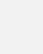


obtained with the MSE of the validation set. Residuals obtained using the AAKR reconstruction method are shown in the upper box, those obtained using the FS reconstruction method are in the middle box, and residuals obtained using the RNN reconstruction method are in the lower box.
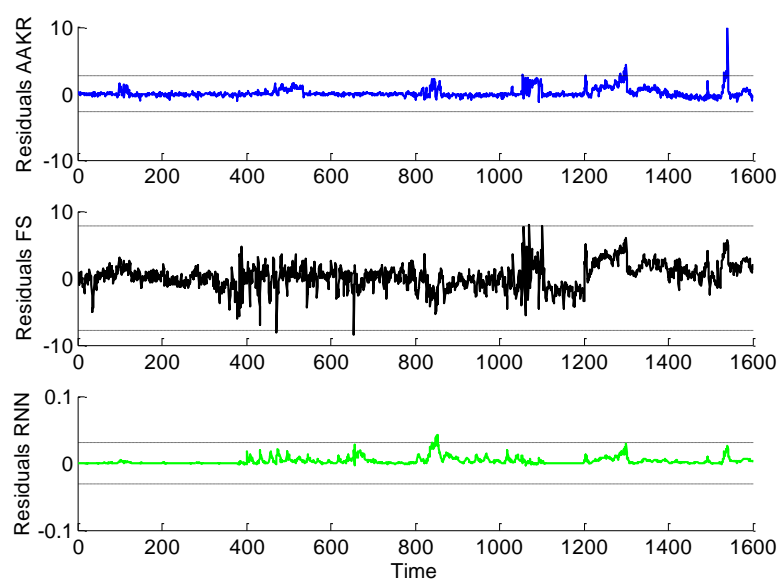

Figure 5. Residuals (continuous line) of the reconstruction of the normal conditions test set with the corresponding thresholds (dashed lines) for the three reconstruction methods: AAKR (upper box), FS (middle box), RNN (lower box). Notice the different scale of the residuals in the lower box.

As shown in Figure 5, RNN is by far the most accurate among the three methods, with threshold much narrower (that is, smaller MSE) than those of FS and AAKR. The test residuals of all three reconstruction methods remain within the region defined by the respective thresholds for almost the entire test set, although residuals of the FS reconstruction show a larger variance with respect to AAKR and RNN. This leads to a larger MSE of the FS reconstruction method, as shown in Table 1.

\begin{tabular}{|c|c|}
\hline Reconstruction method & MSE \\
\hline AAKR & $8.6 \times 10^{-3}$ \\
\hline FS & $7.4 \times 10^{-2}$ \\
\hline RNN & $1.1 \times 10^{-6}$ \\
\hline
\end{tabular}


Table 1. Accuracy of AAKR, FS and RNN in the reconstruction of the test set values.

\subsection{Robustness}

Robustness with respect to the reconstruction of the $j$-th signal is computed by applying Eq. 4 to simulated anomalous conditions such as linear and step drifts that are typical for these signals.

Figures 6 and 7 show the residuals of the reconstructions of the test set for the first and the fourth seasons, respectively, when the $i$-th signal observation is affected by the same linear drift (dotted line): AAKR residuals in the upper box, FS residuals in the middle box, RNN residuals in the lower box.
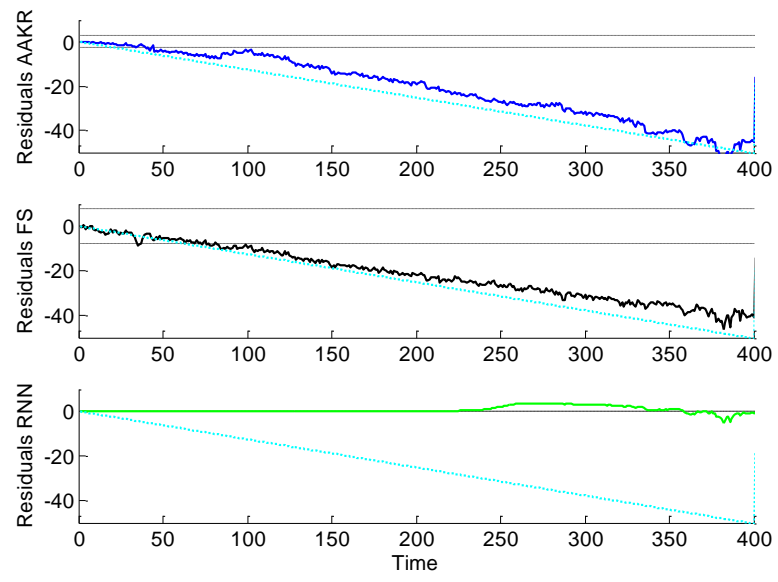

Figure 6. Residuals (continuous line) of the reconstruction of the first season of the test set affected by linear drift (dotted line), with the corresponding thresholds (dashed lines) for the three reconstruction methods: AAKR (upper box), FS (middle box), RNN (lower box). 

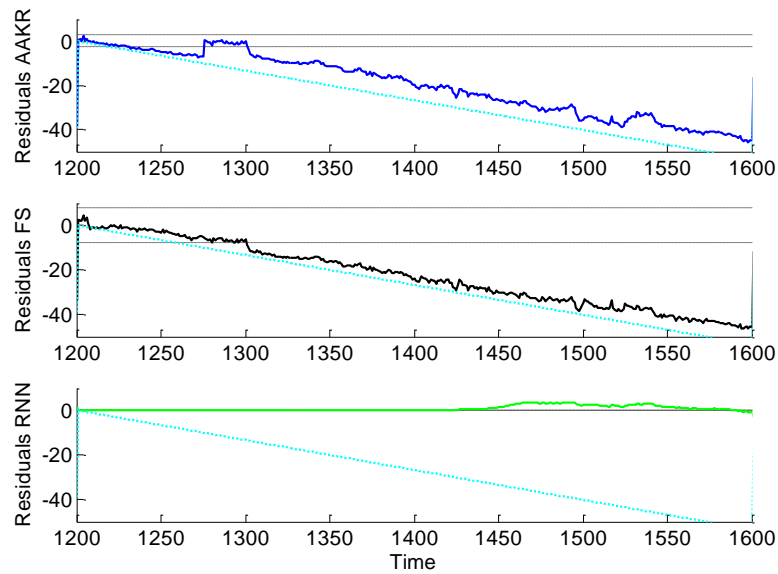

Figure 7. Residuals (continuous line) of the reconstruction of the fourth season of the test set affected by linear drift (dotted line), with the corresponding thresholds (dashed lines) for the three reconstruction methods: AAKR (upper box), FS (middle box), RNN (lower box).

RNN is the worst performing method, whereas FS and AAKR are robust with respect to the linear drift anomaly. In particular, FS tends to perform better than AAKR, as its residuals follow the linear drift more closely and regularly, avoiding multiple alarm triggering (as AAKR would do during the fourth season for measurements at time 1276 and 1302). On the other hand, concerning the fault detection speed, AAKR is less prompt than FS in triggering the alarm. This is due to the fact that FS, even though is more robust, is less accurate and thus has associated an higher threshold (see previous Figure 5). Furthermore, FS tends to delay the anomalous conditions detection due to the need of collecting $L_{t}$ measurements for the comparison of the test trajectory with the reference trajectories. Table 2 summarizes the results in terms of the robustness metric of Eq. (5).

\begin{tabular}{|c|c|}
\hline Reconstruction method & Robustness \\
\hline AAKR & 0.7479 \\
\hline FS & 0.3306 \\
\hline RNN & 13.75 \\
\hline
\end{tabular}

Table 2. Robustness of AAKR, FS and RNN in the reconstruction of the test set affected by a linear drift. 
The residuals obtained in the reconstruction of a test set characterized by the application of a step drift on a signal are shown in Figures 8 and 9 (for the fourth and second seasons, respectively). The obtained results (Table 3) confirm that FS is the most, and RNN the least robust of the reconstruction methods. In both cases of linear and step drifts, the RNN method is not capable of reconstructing the expected value of the signal in normal conditions: the provided reconstruction is indeed very similar to the measurements and from the analysis of the residuals it is not possible to detect abnormal conditions. For these reasons, we consider RNN unsuitable for the detection of failures in this case.

\begin{tabular}{|c|c|}
\hline Reconstruction method & Robustness \\
\hline AAKR & 1.0843 \\
\hline FS & 0.2462 \\
\hline RNN & 13.0428 \\
\hline
\end{tabular}

Table 3. Robustness of AAKR, FS and RNN in the reconstruction of the test set affected by a step drift. 


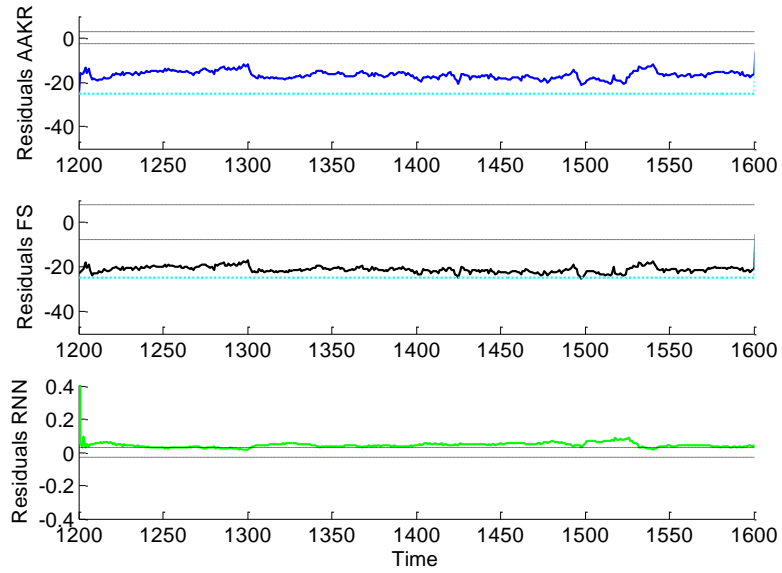

Figure 8. Residuals (continuous line) of the reconstruction of the fourth season of the test set affected by the step drift (dotted line), with the corresponding thresholds (dashed lines) for the three reconstruction methods: AAKR (upper box), FS (middle box), RNN (lower box).
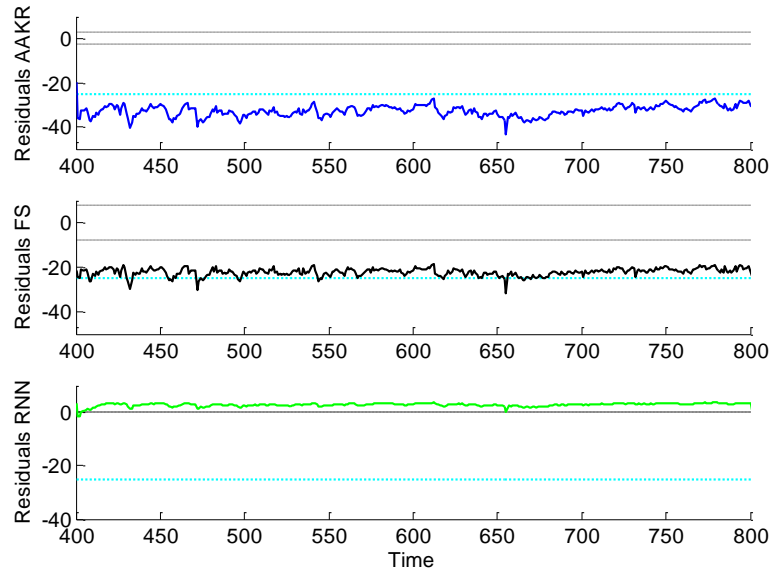

Figure 9. Residuals (continuous line) of the reconstruction of the second season of the test set affected by the step drift (dotted line), with the corresponding thresholds (dashed lines) for the three reconstruction methods: AAKR (upper box), FS (middle box), RNN (lower box). 


\subsection{Spillover}

The spillover effect is evaluated by considering the reconstruction of a signal different from that to which a step drift has been applied. The obtained results are summarized in Table 4: a deviation of the residuals out of the region of normal conditions is a symptom that the reconstruction method is affected by spillover, which can lead to false alarms. Figures 10 and 11 show the residuals of the reconstruction of a signal which is not affected by any step, considering the second and third seasons, respectively and the application of a step on another signal.
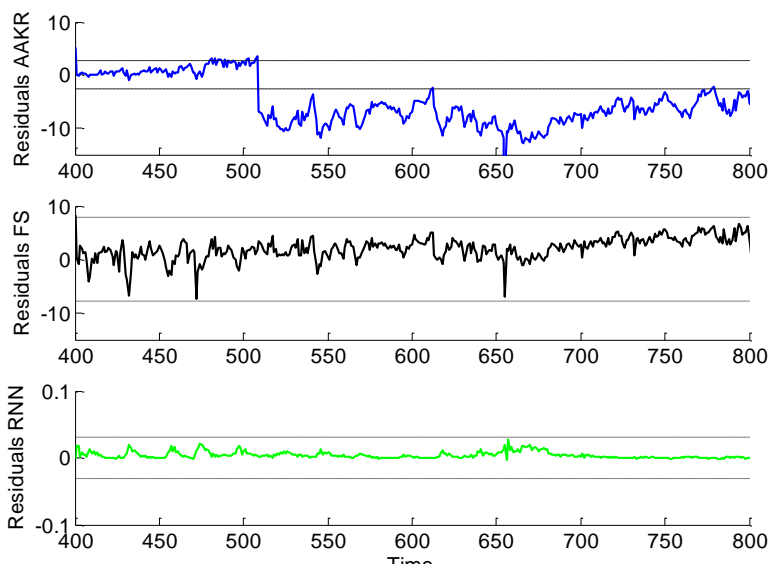

Figure 10. Residuals (continuous line) of the reconstruction of the second season of the test set affected by step drift on a signal different from the plotted one, with the corresponding thresholds (dashed lines) for the three reconstruction methods: AAKR (upper box), FS (middle box), RNN (lower box). 

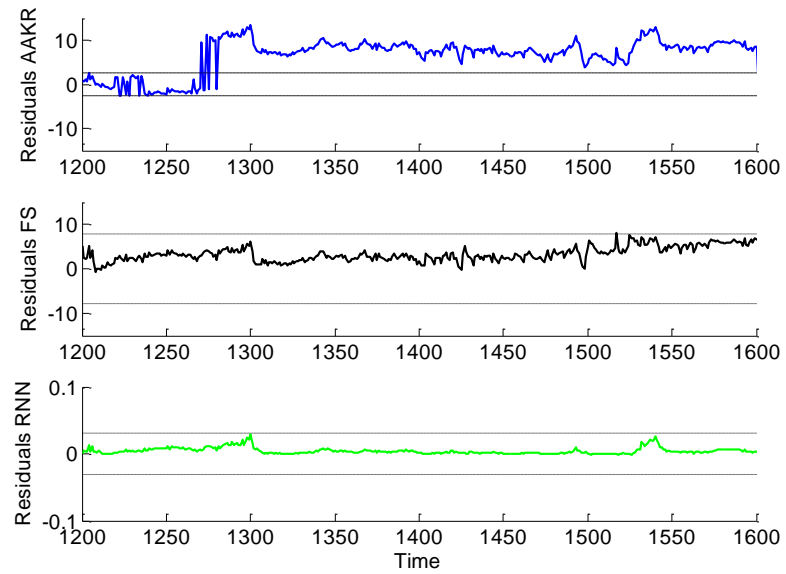

Figure 11. Residuals (continuous line) of the reconstruction of the third season of the test set affected by step drift on a signal different from the plotted one, with the corresponding thresholds (dashed lines) for the three reconstruction methods: AAKR (upper box), FS (middle box), RNN (lower box).

As expected, RNN is not affected by spillover, since (by definition of the model) reconstructions of the $j$-th signal is based exclusively on the $j$-th signal itself, which is not affected by any drift. The AAKR reconstruction method is the most affected by spillover effect which causes the triggering of false alarms on signal $\mathrm{j}$, whereas the residuals provided by the FS always remain below the threshold in the normal conditions region. This difference of the two methods can be explained by the following two observations: 1) since FS is less accurate than AAKR, its failure threshold is higher and thus alarms are more difficult to be triggered 2) among the input of the FS model there are the previous normal condition values of signal $i$ which are, instead not used by the AAKR method and which can reduce the spillover effect.

\begin{tabular}{|c|c|}
\hline Reconstruction method & MSE - Spillover \\
\hline AAKR & 0.7552 \\
\hline FS & 0.2516 \\
\hline RNN & 0 \\
\hline
\end{tabular}

Table 4. Spillover effect of AAKR, FS and RNN in the reconstruction of signal $j$ 


\section{Ensemble}

The comparison of the three methods for signal reconstruction has shown their different capabilities on the data considered. RNN has proven to be the most accurate in the reconstruction of normal conditions and to be not affected by the spillover effect, but it is not robust with respect to the reconstruction of anomalous conditions. Thus, RNN cannot be used as reconstruction method for our fault detection purposes. FS has shown to be satisfactory from the point of view of robustness and spillover effects, but the least accurate, whereas AAKR is the fastest in triggering alarms in case of anomalous conditions. Both FS and AAKR are suitable for our fault detection purposes, although their not completely satisfactory accuracy requires setting high residuals thresholds for the anomalous condition detection. This may cause delays in the triggering of the alarm by the fault detection system.

To overcome this hurdle and further improve reconstruction performance, an ensemble approach can be embraced by way of which a combination of the outcomes of the methods is used so as to benefit from their different capabilities [Baraldi et al., 2010]. The development of an ensemble approach requires the aggregation of the outcomes provided by the three reconstruction methods. A detailed discussion of possible strategies of aggregation (e.g., based on the computation of the simple mean or median of the individual model outcomes) can be found in [Baraldi et al, 2010]. In this work, we consider the median of the reconstruction provided by AAKR, FS and RNN since it allows discarding possible outlying outcomes which could, on the contrary, negatively influence the mean.

Table 5 (columns 1-6) compares the performance of the ensemble with those of FS and AAKR. RNN has not been considered in Table 5 since, due to its low robustness, it cannot be employed alone within our FD system. It is, however, interesting to observe that the ensemble is more accurate and resistant to spillover than the other methods, thanks to the contribution of the RNN reconstructions. Furthermore, with respect to the robustness in the reconstruction of an anomalous condition, the performance of the ensemble is close to that of the AAKR and FS. In practice, in case 
of an anomalous condition, the ensemble outcomes, obtained as the aggregation of the AAKR, FS and RNN outcomes, are not remarkably influenced by the RNN outcomes which are affected by large errors.

In order to further compare the ensemble with the AAKR and FS from the point of view of fault detection performance, an indicator of overall performance, $P_{\text {overall }}$, which aggregates the three metrics of accuracy (MSE), robustness (S) and spillover (A) has been defined according to [Baraldi et al., 2011]:

$$
P_{\text {overal }}=\mathrm{MSE} \times \mathrm{S} \times \mathrm{A}
$$

In Table 5 (last column), it can be noticed that $P_{\text {overal }}$ of the proposed ensemble is more satisfactory than that of FS and AAKR. This confirms that a fault detection system based on an ensemble of the three reconstruction methods considered is capable of overcoming the limitations of each method and of exploiting their strengths, leading to overall satisfactory results with respect to our fault detection purposes.

Future research work will consider the possibility of further improving the reconstruction performance of the ensemble by using other aggregation methods, such as those based on weighted sum of the method outcomes, with weights proportional to the local performance of the methods in the reconstruction of training patterns similar to the test pattern.

Table 5. Reconstruction performance of AAKR, FS, RNN and an ensemble approach

\begin{tabular}{|c|c|c|c|c|c|c|c|c|}
\hline \multirow{2}{*}{$\begin{array}{c}\text { Reconstruction } \\
\text { method }\end{array}$} & \multicolumn{2}{|c|}{ Accuracy } & \multicolumn{2}{c|}{ Robustness } & \multicolumn{2}{c|}{ Spillover } & \multicolumn{2}{c|}{ Overall Performance } \\
\cline { 2 - 9 }$y$ & Value & Ranking & Value & Ranking & Value & Ranking & Value & Ranking \\
\hline AAKR & $8.6 \times 10^{-3}$ & 2 & 1.0843 & 3 & 0.7552 & 3 & $7 \times 10^{-3}$ & 2 \\
\hline FS & $7.4 \times 10^{-2}$ & 3 & $\mathbf{0 . 2 4 6 2}$ & $\mathbf{1}$ & 0.2516 & 2 & $4.5 \times 10^{-3}$ & 3 \\
\hline ENSEMBLE & $\mathbf{6 . 3} \times \mathbf{1 0}^{-3}$ & $\mathbf{1}$ & 0.8712 & 2 & $\mathbf{0 . 1 4 5 1}$ & $\mathbf{1}$ & $\mathbf{0 . 7 9} \times 10^{-3}$ & $\mathbf{1}$ \\
\hline
\end{tabular}




\section{Conclusions}

A comparison of the performance of three reconstruction methods has been presented in the context of fault detection in industrial components. To this aim, real temperature data collected during one year of operation in a rotating machinery has been considered. The comparison has regarded reconstruction methods based on Auto-Associative Kernel Regression (AAKR), Fuzzy Similarity (FS) and Recurrent Neural Network (RNN). The methods have been evaluated from the point of view of accuracy, robustness, spillover effect and speed of anomaly detection.

The results have shown different capabilities and drawbacks of each method. In particular, although RNN has proven to be the most accurate in the reconstruction of normal conditions and to be not affected by the spillover effect, it cannot be effectively used for our fault detection purposes since it is not robust with respect to the reconstruction of anomalous conditions. On the other hand, FS is satisfactory from the point of view of robustness and resistance to the spillover effects, but is the least accurate. Finally, AAKR is the fastest in triggering alarms in case of anomalous conditions, but the least resistant to the spillover effect.

To improve the reconstruction performance, the AAKR, FS and RNN methods have been combined within an ensemble framework. The obtained results have shown that the overall performance of the proposed ensemble is more satisfactory than that of the single methods and is capable of overcoming the limitations of each method while exploiting their strengths.

Future research activity should focus on the optimization of the aggregation of the outcomes of each reconstruction method in the ensemble for further improving accuracy, robustness and resistance to spillover.

\section{Acknowledgments}

The participation of Enrico Zio to this research is partially supported by the China NSFC under grant number 71231001. 


\section{References}

[Al-Bazzaz et al., 2004] H. Al-Bazzaz, X. Z. Wang, New statistical process control chart for batch operations based on independent component analysis, Industrial \& Engineering Chemistry Research, 43, pp. 6731-6741, 2004.

[Angstenberger, 2001] Angstenberger, L., Dynamic Fuzzy Pattern Recognition, International Series in Intelligent Technologies, 17, Kluwer Academic Publishers, 2001.

[Arinton et al., 2012] E. Arinton, S. Caraman, J. Korbicz, Neural networks for modeling and fault detection of the inter-stand strip tension of a cold tandem mill, Control Engineering Practice, 20, pp. 684-694, 2012.

[Baraldi et al., 2010] P. Baraldi, E. Zio, G. Gola, D. Roverso, M. Hoffmann, "Robust nuclear signal Formattato: Non Evidenziato reconstruction by a novel ensemble model aggregation procedure", International Journal of Nuclear Knowledge Management, Vol 4 (1), pp. 34-41, 2010.

[Baraldi et al., 2011] P. Baraldi, R. Canesi, E. Zio, R. Seraoui, R. Chevalier, Genetic algorithmbased wrapper approach for grouping condition monitoring signals of nuclear power plant components, Integrated Computer-Aided Engineering, Volume 18, Issue 3, 2011, Pages 221234.

[Baraldi et al., 2012] P. Baraldi, F. Di Maio, L. Pappaglione, E. Zio, R. Seraoui, Condition Monitoring of Electrical Power Plant Components During Operational Transients, Proceedings of the Institution of Mechanical Engineers, Part O, Journal of Risk and Reliability, 226(6) 568$583,2012$.

[Baraldi et al., 2014] P. Baraldi, F. Di Maio, D.Genini, E. Zio "Reconstruction of missing data in Formattato: Non Evidenziato multidimensional time series by fuzzy similarity" accepted for publication on Applied Soft Computing

[Chetouani, 2006] Y. Chetouani, Fault detection in a chemical reactor by using the standardized innovation, Process Safety and Environmental Protection, 84, pp. 27-32, 2006.

[Coble et al., 2012] J. B. Coble, P.Ramualli, LJ Bond, JW Hines, BR Upadhyaya, Prognostics and Health Management in Nuclear Power Plants: A Review of Technologies and Applications, National Technical Information Service, 2012.

[Di Maio et al., 2013] F. Di Maio, P. Baraldi, E. Zio, R. Seraoui, Fault Detection in Nuclear Power Plants Components by a Combination of Statistical Methods, accepted, IEEE Transaction, December 2013

[Elman, 1990] J. Elman, Finding structure in time, Cognitive Science 14, 179, 1990. 
[Far et al., 2009] R. Far, H. Davilu, V. Palade, C. Lucas, Model-based fault detection and isolation of a steam generator using neuro-fuzzy networks, Neurocomputing, 72, pp. 2939-2951, 2009.

[Gabrijel et al., 2003] I. Gabrijel, A. Dobnikar, On-line identification and reconstruction of finite automata with generalized recurrent neural networks, Neural Networks, 16, pp. 101-120, 2003.

[Garvey et al., 2006] D.R. Garvey, J. W. Hines., The Development of a Process and Equipment Monitoring (PEM) Toolbox and its Application to Sensor Calibration Monitoring, Quality and Reliability Engineering International, 22, pp. 1-13, 2006.

[Hines et al., 1996] J. W. Hines, D. J. Wrest, R. E. Uhrig, Plant Wide Sensor Calibration Monitoring, In Proceedings of the 1996 IEEE International Symposium on Intelligent Control, Dearborn, Michigan. IEEE, Piscataway, New Jersey, pp. 378-383. September 15-18, 1996.

[Hines et al., 2008] J. W. Hines, D. Garvey, R. Seibert, A. Usynin, Technical Review of on-line monitoring techniques for performance assessment, Volume 2: Theoretical Issues, Office of Nuclear Regulatory Research, 2008.

[Luh et al., 2011] G. C. Luh, C.Y. Lin, PCA based immune networks for human face recognition Applied Soft Computing Journal, 11 (2), pp. 1743-1752, 2011.

[Ma et al.,2011] J. Ma, J. Jiang, Applications of fault detection and diagnosis methods in nuclear power plants: a review, Progress in Nuclear Energy, 53, 2011.

[Pham et al., 1996] D. T. Pham, X. Liu, Training of Elman networks and dynamic system modeling, International Journal of System Science, vol 27(2), pp. 221-226, 1996

[Reifman, 1997] J. Reifman, Survey of artificial intelligence methods for detection and identification of component faults in nuclear power plants, Nuclear Technology, 119, pp. 7697, 1997.

[Rumelhart et al., 1986D. E. Rumerlhart, G. E. Hinton, R. J. Williams, Learning representations by back-propagating errors, Nature, 323, 533-536, 1986); doi:10.1038/323533a0

[S. Seker et al., 2003] S. Seker, E. Ayaz, E. Türkan, Elman's recurrent neural network applications to condition monitoring in nuclear power plant and rotating machinery, Engineering Applications of Artificial Intelligence 16, pp. 647-656, 2003.

[Wang et al., 2005] S. Wang, J. Qin, Sensor fault detection and validation of VAV terminals in air conditioning systems, Energy Conversion and Management, 46, pp. 2482-2500, 2005.

[Yu et al., 2006] C. Yu, B. Su, Eliminating false alarms caused by fault propagation in signal validation by sub-grouping, Progress in Nuclear Energy, 48, pp. 317-379, 2006.

[Zavaljevski et al., 2000] N. Zavaljevski, K. Gross, Sensor fault detection in nuclear power plants using multivariate state estimation technique and support vector machines, The Third 
International Conference of the Yugoslav Nuclear Society YUNSC, pp. 1-11, Belgrade, Yugoslavia, 2000.

[Zhang et al., 2011] Z. Zhang; H. Xu; R. Martin; C. Liu, Inferential Model for Linear Regression, Pakistan Journal of Statistics and Operation Research;7(2 SPECIAL ISSUE):413-432, 2011.

[Zhao et al., 2013] W. Zhao, T. Tao, Z. S. Ding, E. Zio, A dynamic particle filter-support vector regression method for reliability prediction, Reliability Engineering and System Safety 119 (2013) 109-116.

[Zio et al., 2010a] E. Zio, F. Di Maio, A data-driven fuzzy approach for predicting the remaining useful life in dynamic failure scenarios of a nuclear system, Reliability Engineering and SystemSafety, 95, 2010.

[Zio et al., 2010b] E. Zio, F. Di Maio, M. Stasi, A Data Driven Approach for Predicting Failure Scenarios in Nuclear Systems, Annual of Nuclear Energy, 37, 482-491, 2010.

[Zio et al., 2010c] E. Zio, F. Di Maio, A fuzzy similiarity-based Method for Failure Detection and Recovery Time Estimation, International Journal of Performability Engineering, 6, No. 5, 2010. [Zio et al., 2011] E. Zio, P. Baraldi, W. Zhao, Confidence in signal reconstruction by the Evolving Clustering Method, 2011 Prognostics and System Health Management Conference, PHMShenzhen, 24-25 May, 2011.

[Zio et al., 2012] E. Zio, F. Di Maio, Fatigue Crack Growth Estimation by Relevance Vector Machines, Expert Systems with Applications, Volume 39, pp. Volume 39, Issue 12, Pages 10681-10692 http://dx.doi.org/10.1016/j.eswa.2012.02.199. 